early childhood. These patients do not seem to owe their decline to persistent infection or to reinfection. Pyelonephritis of pregnancy is not often the primary cause of much renal damage, though it can unmask and seriously worsen preexisting disease.

Deaths from chronic pyelonephritis in childhood are exceptional and under the age of 40 rare. After this they become more frequent, especially in women, there being, it is thought, a disproportionate number of deaths from chronic pyelonephritis in women between 50 and 70 years of age. Most of these patients are unlikely to be suitable for surgical correction of hypertension or to be accepted in a renal dialysis or transplant programme. McGregor believes that the extent of serious renal disease in early adult life as a result of childhood urinary infection has been exaggerated, though a few striking tragedies occur. Nevertheless, longevity, especially in women, may be considerably reduced.

The diagnosis of chronic pyelonephritis presents formidable difficulties. Beeson ${ }^{3}$ considers there are no direct symptoms, and that physical findings likewise are normal except for those caused by renal insufficiency. Microscopical study of formed elements in the urine may disclose surprisingly little. Proteinuria is variable and never large in amount; bacteriuria alone is not diagnostic, while patients with unquestionable chronic pyelonephritis may have negative urine cultures. The provocation of urinary leucocyte excretion by administering intravenous pyrogen ${ }^{7}$ or prednisolone ${ }^{8}$ has proved helpful, but unfortunately is not specific for pyelonephritis, similar findings being obtained in some patients with glomerulonephritis. Radiology seems the most helpful aid to diagnosis, the essential features being asymmetry and irregularity of the renal outlines, with bluntitg of one or more calices and diminished cortical thickness at the corresponding sites.

From the morbid anatomist's point of view Heptinstall ${ }^{6}$ has emphasized the coarse irregular scarring of chronic pyelonephritis, there often being unequal reduction in the size of the two kidneys, in contrast to the symmetrical contraction of chronic glomerulonephritis and most other forms of shrunken kidney. The depressed, scarred, cortical areas of the renal surface lie opposite dilated and club-shaped calices, with intervening areas appearing normal. Heptinstall stresses the importance of the gross examination of the kidney because of the largely nonspecific microscopical appearances of the parenchymal response to infection. Renal biopsy is of doubtful value, if it is not positively misleading, because of the small sample obtained, the patchy nature of the disease, and the lack of certainty of diagnosis on histological criteria.

The particular emphasis in these recent reviews has been criticism of the indiscriminate diagnosis of chronic nyelonephritis. Evaluation of treatment also is made difficult by the paucity of knowledge of the natural history of urinary infections in childhood and early adult life. McGregor is critical of many of the reports of benefit from long-term antibiotic therapy or from surgical correction of ureterovesical reflux, because of the absence of valid control groups in most of these studies.

Pickering's summary ${ }^{5}$ of the present situation of chronic pyelonephritis seems apt: "The disease is as yet by no means clearly defined. The problems of its determination, its diagnosis, its relationship to hypertension and its treatment are still largely sub judice."

1 McGregor, M., Archives of Disease in Childhood, 1970, 45, 159.

2 Heptinstall, R. H., Pathology of the Kidney. Boxton, Little, Brown, 1966.

3 Beeson, P. B., in Renal Disease, ed. D. A. K. Black, 2nd edn., p. 382. Oxford, Blackwell, 1967.
- Milne, M. D., in The Human Adrenal Cortex, ed. A. R. Currie, T. Symington, and J. K. Grant, p. 493. Edinburgh, Livingstone, 1962.

Pickering, G. W., High Blood Pressure, 2nd ledn., London, Churchill, 1968.

- Heptinstall, R. H., in Renal Disease, ed. D. A. K. Black, 2nd edn., p. 350. Oxford, Blackwell, 1967.

Pears, M. A., and Houghton, B. J., Lancet, 1959, 2, 1167.

Little, P. J., and de Wardener, H. E., Lancet, 1962, 1, 1145.

\section{Ups and Downs in the Health Service}

During the decade up to 1969 the numbers of medical staff in the Health Service fluctuated, going up in some sections of it and down in others. ${ }^{1}$ The total number of hospital beds actually fell by $4 \%$. What did rise indisputably was the amount spent on it. In Great Britain as a whole this figure more than doubled, from $£ 707 \mathrm{~m}$. to $£ 1,573 \mathrm{~m}$., though what this means in terms of real money (if such a thing still exists) is hard to say. Despite inflation the increase does seem to be substantial even though the antiquated corners of the Health Service that still remain show it to have fallen below the nation's real needs.

The number of hospital beds in Britain fell from 548,676 in 1959 to 528,917 in 1969 . The bulk of the vanishing beds were in psychiatric hospitals, their closure being the consequence of the greater emphasis on treating these patients at home and as outpatients. But in the nonpsychiatric hospitals there was also a diminution of the number of beds amounting to some 2,000. Nor was it only beds that became fewer: hospitals did so too. One hundred and seventy of them disappeared, comprising a reduction of about $6 \%$. But within the general reduction in the number of hospitals, and in contrast to the loss of psychiatric beds, the number of psychiatric hospitals actually rose from 374 to $404(8 \%)$. A notable feature of this changeover was the closure of 20 psychiatric hospitals each containing more than 2,000 beds. At the same time more smaller hospitals were opened.

While hospital beds were declining in number during this decade the medical staff in the hospitals increased by $37 \%$ in terms of whole-time equivalent appointments. Though nearly all specialties shared in this expansion, paediatric surgery and traumatic and orthopaedic surgery are notable for doubling in size. The total number of consultants in England and Wales (for which figures are available) increased from 5,322 in 1959 to 7,763 in 1969 $(46 \%)$, while senior registrars and registrars went up by $59 \%$ from 3,718 to 5,898 . The net result of all these changes was something as dear to the modern economist as it would have been to Florence Nightingale-higher turnover. Many more inpatients and outpatients were treated per year at the end of the decade than at its beginning-some $30 \%$ - and the average time spent in hospital was reduced from 38 days to 26 days (32\%).

The story in general practice is very different. Instead of the large increase in staff that the hospitals enjoyed the total number of general practitioners was lower in Great Britain (and in England and Wales separately) at the end of the decade than in 1959. They began at just over 25,000 and ended at just over 24,000 . The number of unrestricted principals was also slightly lower, falling from 22,820 to 22,727 . The nadir was reached in $1966-7$, and thereafter the increase has been steady among principals 
and also trainees. Comparable figures for the public health service are not given.

To economists and to hospital administrators, to politicians and to members of local health atthoricies si:ch figures as these rose a variety of problems in the providing of medical care for the population. But what do they mean in terms of health for better or worse? Here it is extraordinarily difficult to see any direct relationship between the main statistics of mortality and morbidity and the figures showing that more or fewer doctors are practising, beds are in use, or drugs are prescribed. Though 1969 showed the lowest infant mortality and stillbirth rates ever recorded in England and Wales, the expectation of life at birth was lower than in many other countries. ${ }^{2}$ In no fewer than 17 countries were the males better off than in England and Wales in that respect, and in five countries the females were.

The fact is that Britain will have to work hard to improve its health services if it is going to keep in the same league as other technically advanced countries. And medical care, like any other service for the whole community, reflects the general state of the nation's economy. If that is undermined, the consequences can only be harmful for the nation's health.

1 Department of Hralth and Social Security, Digest of Health Statistics. London, H.M.S.O., 1971.

Departm nt of Health and Social Security, On The State of The Pub lic Health. London, H.M.S.O., 1970.

\section{Penicillin and the Mouth Flora}

In long-term treatment for preventing recurrences of rheumatic fever sulphonamides gave place to penicillin mainly because sulphonamide resistance in group A streptococci became increasingly common. Sulphadiazine is nevertheless still sometimes used, and it would be interesting to know how frequent such resistance is now. Have haemolytic stı eptococci, like gonococci, reverted to sulphonamide sensitivity in consequence of much-reduced exposure to these drugs?

There are several ways of administering penicillin for this purpose, and their relative merits were discussed in these columns four years ago. ${ }^{1}$ It then seemed clear from the studies of $\mathrm{H}$. F. Wood and his colleagues ${ }^{2}$ that a monthly injection of benzathine penicillin was by far the most effective regimen. In groups of patients given oral penicillin, benzathine penicillin, and sulphadiazine the streptococcal infection rates per 100 patient years were $20 \cdot 7,6 \cdot 1$, and 24 , and the recurrence rates for rheumatic fever $5.5,0.4$, and 2.8 respectively. There was also evidence in another study that a single daily oral dose of benzyl penicillin had an inadequate, if indeed any, effect, and it was suggested that in oral treatment two daily doses are advisable, preferably of penicillin $\mathrm{V}$, which is much better and more regularly absorbed.

An effect of such treatment which should be borne in mind is that on the streptococcal flora of the mouth. That penicillinresistant strains of Streptococcus viridans appear in the mouth of children treated with penicillin was first reported over 20 years ago by $\mathrm{E}$. Krumwiede. ${ }^{3}$ The doses given for rheumatic fever prophylaxis were very small, and all the strains of streptococci were inhibited by 1 unit per $\mathrm{ml}$. More recent studies date from that of L. P. Garrod and P. M. Waterworth, who reported two cases in which endocarditis due to highly penicillin-resistant streptococci followed dental extraction during penicillin treatment. They cultivated saliva from patients receiving penicillin, most of them for rheumatic fever prophylaxis, and round the great majority of the streptococci to be inhibited only by $2-32$ units $/ \mathrm{ml}$ of penicillin, whereas most of those from controls were inhibited by 0.06 unit $/ \mathrm{ml}$ or less. These findings have been confirmed by workers in both Great Britain and the United States. It is now recognized that when penicillin is administered either orally or parenterally its excretion in the saliva immediately suppresses the sensitive streptococcal flora and enables a probably minute proporticn of resistant organisms to multiply in their place. In the original study cited, ${ }^{4}$ streptococci were found to have disappeared one day after the start of treatment with therapeutic doses of penicillin $\mathrm{V}$, to be replaced by resistant organisms on day 2 . Hence, if cover is to be given for dental extraction, the first dose of penicillin should precede the operation only by the length of time required to attain the maximum concentration in the blood. Reversion to sensitivity when treatment is stopped has also been studied, with particular thoroughness by Katherine Sprunt and her colleagues. ${ }^{5}$ Though the proportion of resistant streptococci soon falls, they may persist in diminished numbers for weeks.

The latest study of this subject is by W. H. Spencer and his colleagues. ${ }^{6}$ They cultivated gingival swabs from rheumatic patients receiving sulphadiazine or penicillin and from controls, and determined the penicillin sensitivity of the organisms found. This study differs from others in that the entire (aerobic) oral flora was examined. Thus in one group of 54 resistant organisms only half were Str. viridans and the remainder Staphylococcus epidermidis or "micrococci." A somewhat cumbersome cultural method was used, involving replica plating. This was designed "to limit bacterial interference on the culture plates," a difficulty which does not seem to have deterred other investigators or to have interfered with their obtaining apparently significant results. The results in general confirm the findings of others, but are less clear-cut. Thus some resistant organisms were found in controls (how many of these were Str. viridans is not stated), and the highest percentage with such organisms among those treated with penicillin was only 42 . In other studies this proportion has been higher, and it is not clear whether this difference is attributable to the cultural method used or perhaps to failure on the patients' part to take their tablets, a factor referred to in the American literature as "fidelity of prophylaxis" or "penicillin compliance." Among 57 patients having oral penicillin studied by P. Y. Paterson and Gloria M. Madden ${ }^{7} 38$ had resistant streptococci in their mouths, but when urines were tested for penicillin 25 out of 29 showing good compliance had resistant organisms but only 4 out of 10 with low compliance. Spencer and his colleagues confirm a finding of these authors, that resistant organisms are not found in patients treated with benzathine penicillin. The very low concentrations of penicillin so produced are evidently enough to keep the highly sensitive haemolytic streptococcus away but not enough to affect the resistance of other species.

How are patients on oral prophylaxis to be covered for dental extraction? Vancomycin, which is troublesome to administer, or a large dose of penicillin reinforced with streptomycin, has been suggested. R. A. Tozer, S. Boutflower, and W. A. Gillespie ${ }^{8}$ found that resistant streptococci from such patients are susceptible to the bactericidal action of cephaloridine and suggest a single $0.5 \mathrm{-g}$ dose, perhaps followed by erythromycin, which has a similar effect. According to R. M. Stirland and Nina Shotts ${ }^{9}$ such streptococci are not 